\title{
Projective Center Point and Tverberg Theorems
}

\author{
Roman Karasev • Benjamin Matschke
}

Received: 24 November 2012 / Revised: 28 November 2013 / Accepted: 10 May 2014 /

Published online: 25 June 2014

(C) Springer Science+Business Media New York 2014

\begin{abstract}
We present projective versions of the center point theorem and Tverberg's theorem, interpolating between the original and the so-called "dual" center point and Tverberg theorems. Furthermore we give a common generalization of these and many other known (transversal, constraint, dual, and colorful) Tverberg type results in a single theorem, as well as some essentially new results about partitioning measures in projective space.
\end{abstract}

Keywords Center point theorem $\cdot$ Tverberg's theorem $\cdot$ Measure partitions

Mathematics Subject Classification $52 \mathrm{~A} 35 \cdot 52 \mathrm{C} 35$

\section{Introduction}

In this paper we focus on two classical topics in discrete geometry: The center point theorem from Neumann [20] and Rado [22] (see also Grünbaum [11]) and Tverberg's theorem [24]. Many deep generalizations of these classical results have

\author{
R. Karasev \\ Department of Mathematics, Moscow Institute of Physics and Technology, \\ Institutskiy per. 9, Dolgoprudny 141700, Russia \\ R. Karasev \\ Institute for Information Transmission Problems RAS, Bolshoy Karetny per. 19, \\ Moscow127994, Russia \\ e-mail: r_n_karasev@mail.ru \\ B. Matschke \\ Max Planck Institute for Mathematics, Bonn, Germany \\ e-mail: matschke@mpim-bonn.mpg.de


been made in the last three decades, starting from the topological generalization by Bárány et al. [1]. A good review on this topic and numerous references are given in Matoušek's book [19]. After this book was published, new achievements were made by Hell [12,13], Engström [9] and Engström-Norén [10], Karasev [16], and Blagojević et al. [3,4], establishing "constrained", "dual", and "optimal colorful" Tverberg type theorems. In this paper we present a common generalization of all of these results, which naturally lives in projective space.

Center point theorems First, let us review the center point theorem and its dual. In $\mathbb{R}^{d}$, a hyperplane $H$ is an affine subspace of codimension one, and a closed halfspace is the closure of a connected component of the complement of some hyperplane.

Theorem 1.1 (Center point theorem [20,22]) For any finite set $X \subset \mathbb{R}^{d}$ there exists a point $W \in \mathbb{R}^{d}$ such that any closed halfspace containing $W$ contains at least $\left\lceil\frac{|X|}{d+1}\right\rceil$ points of $X$.

Any such $W$ is called a center point for the given point set $X$. The set of all center points for a given point set $X$ is a convex compactum. The first author [16] proved a dual center point theorem, which states the following.

Theorem 1.2 (Dual center point theorem [16]) For any family of $n$ hyperplanes in general position in $\mathbb{R}^{d}$ there exists a point $W$ such that any ray starting at $W$ intersects at least $\left\lceil\frac{n}{d+1}\right\rceil$ hyperplanes.

Here the use of the adjective "dual" is rather descriptive, it does not mean that Theorems 1.1 and 1.2 are equivalent under projective duality. Thus it is interesting to dualize Theorem 1.2 projectively and compare it with the original center point theorem. First let us make a useful definition:

Definition 1.3 A hyperplane in $\mathbb{R} P^{d}$ is a projective subspace of codimension one. Any two distinct hyperplanes $H_{1}$ and $H_{2}$ partition $\mathbb{R} P^{d}$ into two parts. In this paper, we always consider the parts as being closed. If $H_{1}$ and $H_{2}$ coincide then we define $H_{1}=H_{2}$ as one part and the whole $\mathbb{R} P^{d}$ as the other (such that the parts change continuously in the Hausdorff metric when perturbing $H_{1}$ and $H_{2}$ ).

The projective dual of Theorem 1.2 reads: Assume that $X$ is a family of $n$ points in $\mathbb{R} P^{d}$ and $V \in \mathbb{R} P^{d}$ is another point such that the family $X \cup V$ is in general position. Then there exists a hyperplane $W \subseteq \mathbb{R} P^{d}$ such that together with any hyperplane $H_{V}$ containing $V$ it partitions $\mathbb{R} P^{\bar{d}}$ into two parts each containing at least $\left\lceil\frac{n}{d+1}\right\rceil$ points of $X$. From the proof of this theorem we can assure that $W$ does not contain $V$; however if we omit the general position assumption then the theorem remains true by a compactness argument but $W$ may happen to contain $V$.

Our first result interpolates between Theorem 1.1 and the projective dual of Theorem 1.2, which appear as the special cases when $V$ is the hyperplane at infinity and when $V$ is a point, respectively:

Theorem 1.4 (Projective center point theorem) Suppose that $V \subset \mathbb{R} P^{d}$ is a projective subspace of dimension $v$ and $X$ is a finite point set with $|X|=n$. Put $w:=d-v-1$ and 


$$
r=\left\lceil\frac{n}{(d-v)(d-w)+1}\right\rceil .
$$

Then there exists a projective subspace $W \subset \mathbb{R} P^{d}$ of dimension $w$ such that any pair of hyperplanes $H_{V} \supseteq V$ and $H_{W} \supseteq W$ partitions $\mathbb{R} P^{d}$ into two parts each containing at least $r$ points of $X$.

If we require the general position assumption that no $r$ points of $X$ together with $V$ are contained in a hyperplane, then $W$ may be chosen disjoint from $V$.

Remark 1.5 A word about where the numbers come from: First, $w=\operatorname{dim} W$ is chosen such that the linearizations of $V$ and $W$ in $\mathbb{R}^{d+1}$ are of complementary dimension. For smaller $w$ we cannot prove anything interesting, since no matter how one chooses $W$ in this case there is a hyperplane containing both $V$ and $W$. For larger $w$ we will be able to prove more, namely that one can treat several point sets at the same time, see Sect. 5. The number $r$ is best possible for $v=d-1$ (for this consider $n$ points that are distributed as uniformly as possible at the vertices of a $d$-simplex), but for general $v$ this is not clear.

The ordinary center point theorem $[20,22]$ is usually stated for measures, which follows from the discrete version by an approximation argument. Here is the corresponding version of Theorem 1.4:

Theorem 1.6 (Projective center point theorem for measures) Suppose that $V \subset \mathbb{R} P^{d}$ is a projective subspace of dimension $v$ and $\mu$ is a Borel probability measure on $\mathbb{R} P^{d}$. Then there exists a projective subspace $W \subset \mathbb{R} P^{d}$ of dimension $w:=d-v-1$ such that any pair of hyperplanes $H_{V} \supseteq V$ and $H_{W} \supseteq W$ partitions $\mathbb{R} P^{d}$ into two parts $P_{1}$ and $P_{2}$ so that

$$
\mu\left(P_{1}\right), \mu\left(P_{2}\right) \geq \frac{1}{(d-v)(d-w)+1} .
$$

Remark 1.7 If $\mu$ is absolutely continuous then no solution $W$ intersects $V$ : Otherwise there is a hyperplane $H$ containing both $V$ and $W$. Choosing $H_{V}=H_{W}=H$ yields a partition one of whose parts is $H$, which is a zero set.

Tverberg theorems. The same can be done with Tverberg's classical result and it's dual version:

Theorem 1.8 (Tverberg's theorem [24]) Any finite set $X \subseteq \mathbb{R}^{d}$ of $(d+1)(r-1)+1$ points can be partitioned into $r$ parts $X_{1}, \ldots, X_{r}$ whose convex hulls have a point in common.

Theorem 1.9 (Dual Tverberg theorem [16]) Suppose $r$ is a prime power and $\mathcal{F}$ is a family of $(d+1) r$ hyperplanes in general position in $\mathbb{R}^{d}$. Then $\mathcal{F}$ can be partitioned into $r$ subfamilies $\mathcal{F}_{1}, \ldots, \mathcal{F}_{r}$ of $d+1$ hyperplanes each, so that the simplexes formed by the subfamilies have a common interior point.

Here we use the fact that any $d+1$ hyperplanes in general position partition $\mathbb{R}^{d}$ into several parts, only one of which is a bounded simplex. 
Our second result interpolates between (the prime power case of) Theorem 1.8 and the projective dual of Theorem 1.9, which appear as the special cases when $V$ is the hyperplane at infinity and when $V$ is a point, respectively:

Theorem 1.10 (Projective Tverberg theorem) Suppose that $V \subset \mathbb{R} P^{d}$ is a projective subspace of dimension $v$ and $X$ is a finite point set with $|X|=(D+1)(r-1)+1$, where $D:=(d-v)(d-w), w:=d-v-1$, and $r$ is a prime power.

Then there exists a projective subspace $W \subset \mathbb{R} P^{d}$ of dimension $w$ and a partition of $X$ intor subfamilies $X_{j}(j=1, \ldots, r)$ such that any pair of hyperplanes $H_{V} \supseteq V$ and $H_{W} \supseteq W$ partitions $\mathbb{R} P^{d}$ into two parts so that each part has nonempty intersection with every $X_{j}$.

Remark 1.11 An additional general position assumption "no $r$ points of $X$ together with $V$ are contained in a hyperplane" will again guarantee that $W$ may be chosen disjoint from $V$.

Remark 1.12 The number of partitions of $X$ into $r$ subfamilies such that there exist a $W$ as in the theorem can be bounded from below as in Vučić-Živaljević [28] (if $r$ is a prime) and in Hell [12] (for $r=p^{\ell}$ ) by

$$
\frac{1}{(r-1) !}\left(\frac{r}{\ell+1}\right)^{\lceil(r-1)(d+1) / 2\rceil} .
$$

Remark 1.13 There is also a colorful extension as in Blagojević et al. [3] (compare with Remark 4.4) and "constraint Tverberg"-extensions as in Hell [13] and EngströmNorén [10]. In the proof we only need to invoke those extensions in place of the topological Tverberg theorem.

Contents of this paper. The proofs of Theorems 1.4 and 1.10 are given in Sects. 2 and 3, respectively. In Sect. 4 we state a very general theorem incorporating almost all that we know about (dual, transversal, constrained, colorful) Tverberg type theorems. In particular this implies a projective center transversal theorem, which generalizes Theorem 1.6, see Sect. 5. Exchanging quantors can possibly lead to other interesting theorems. As an instance of this we prove another projective Tverberg theorem and a transversal generalization in Sects. 6 and 7.

Open problems. Some of our theorems need technical assumptions in order to assure that certain topological obstructions do not vanish and such that we are able to prove that. These include the usual prime power assumption in Tverberg type theorems, as well as more specific assumptions in the results of Sects. 6 and 7. It would be very interesting to know in which cases these assumptions are necessary and in which cases they can be avoided.

Moreover, Sects. 6 and 7 already indicate that interesting versions and generalizations of our projective center point and Tverberg theorems might be obtained by exchanging quantors and by including additional subspaces on top of $V$ and $W$, also in connection to ham sandwich type mass partition theorems. Is there a natural generalization incorporating all results of this paper? 


\section{Proof of the Projective Center Point Theorem}

We will need the topological center point theorem from the first author [17]:

Theorem 2.1 (Topological center point theorem [17]) Suppose $n=(d+1)(r-1)+1$, $r \geq 1$, and let $\Delta^{n-1}$ be the $(n-1)$-dimensional simplex, and let $M$ be a metric space of covering dimension d. Suppose $f: \Delta^{n-1} \rightarrow M$ is a continuous map. Then

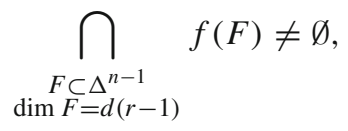

where the intersection is taken over all faces of dimension $d(r-1)$, that is, of codimension $r-1$.

Deo [5] extended this theorem to arbitrary Hausdorff spaces $M$ of covering dimension $d$. We will only use it for $M=\mathbb{R}^{d}$, and this particular case follows from Theorem 3.1. The restriction that $r$ is a prime power can be avoided by a trick with taking points with multiplicities, see [17, Sect. 4] for the details.

Proof of Theorem 1.4 Suppose we are given $V \subset \mathbb{R} P^{d}$ and $X \subset \mathbb{R} P^{d}$ as in Theorem 1.4. Write $X=\left\{\left[x_{1}\right], \ldots,\left[x_{n}\right]\right\}$ with $x_{i} \in \mathbb{R}^{d+1}$, where $\left[x_{i}\right]$ denotes the line through $x_{i} . V$ corresponds to a linear subspace of $\mathbb{R}^{d+1}$ of dimension $v+1$, and by polarity, to a linear subspace $\widehat{V} \subset\left(\mathbb{R}^{d+1}\right)^{*}$ of dimension $d-v$. Similarly, instead of constructing the desired $W \subset \mathbb{R} P^{d}$ of dimension $w:=d-v-1$, we will construct the corresponding linear subspace $\widehat{W} \subset\left(\mathbb{R}^{d+1}\right)^{*}$ of dimension $d-w$.

Consider the standard simplex $\Delta^{n-1}$ with vertices indexed by $\{1, \ldots, n\}$. A point $p \in \Delta^{n-1}$ is an $n$-tuple of nonnegative reals $p=\left(p_{1}, \ldots, p_{n}\right)$ that sum up to 1 , which can be regarded as a probability distribution on $X$. For any $p \in \Delta^{n-1}$ and $\varepsilon>0$ we define the following quadratic form on $\left(\mathbb{R}^{d+1}\right)^{*}$ :

$$
q_{p, \varepsilon}(\varphi)=\sum_{i=1}^{n} p_{i} \varphi\left(x_{i}\right)^{2}+\varepsilon\|\varphi\|^{2}
$$

where $\|\cdot\|$ is the standard norm on $\left(\mathbb{R}^{d+1}\right)^{*}$. We would prefer to put $\varepsilon=0$, but we have to consider positive $\varepsilon$ in order to make sure that $q_{p, \varepsilon}$ is positive definite for any $p \in \Delta^{n-1}$.

Define $\widehat{W}_{p, \varepsilon} \subset\left(\mathbb{R}^{d+1}\right)^{*}$ to be the orthogonal complement of $\widehat{V}$ with respect to the scalar product defined by $q_{p, \varepsilon}$. Obviously $\widehat{W}_{p, \varepsilon}$ depends continuously on $p \in \Delta^{n-1}$ and $\varepsilon>0$ and $\widehat{W}_{p, \varepsilon}$ always has trivial intersection with $\widehat{V}$. Note that the space of all possible linear subspaces $\widehat{W}$ of dimension $d-w$ having trivial intersection with $\widehat{V}$ is a Schubert cell in the Grassmannian $G(d+1, d-w)$, which is homeomorphic to $\mathbb{R}^{D}, D=(d-v)(d-w)$. Thus for a fixed $\varepsilon>0$ we get a map

$$
f: \Delta^{n-1} \rightarrow \mathbb{R}^{D}
$$

that sends $p$ to $\widehat{W}_{p, \varepsilon}$. 
Now we invoke Theorem 2.1 and obtain that the intersection of the images of all faces of codimension $r-1=\left\lceil\frac{n}{D+1}\right\rceil-1$ in $\Delta^{n-1}$ is non-empty.

Thus in our situation we find a subspace $\widehat{W}_{\varepsilon}$ with the following property: For any face $F \in \Delta^{n-1}$ of dimension at least $n-r$ (i.e. spanned by $n-r+1$ vertices of $\Delta^{n-1}$ ) there exists $p_{F, \varepsilon} \in F$ such that $\widehat{W}_{\varepsilon}=\widehat{W}_{p_{F, \varepsilon}, \varepsilon}$. Recall that $r=\left\lceil\frac{n}{D+1}\right\rceil$.

As simplices and Grassmannians are compact we can choose a sequence $\varepsilon_{m} \rightarrow+0$ and corresponding points $p_{F, \varepsilon_{m}}$ such that the respective $\widehat{W}_{\varepsilon_{m}}$ converge to some $\widehat{W}$ in the Grassmannian topology, the points $p_{F, \varepsilon_{m}}$ tend to some points $p_{F}$, and so do the quadratic forms $q_{p_{F, \varepsilon_{m}}, \varepsilon_{m}} \rightarrow q_{F}$. By continuity, $q_{F}=q_{p_{F}, 0}$.

Now we prove that this $\widehat{W}$ fulfills the statement of the theorem. Consider hyperplanes $H_{V} \supseteq V$ and $H_{W} \supseteq W$. They correspond to linear functionals $\varphi_{V} \in \widehat{V}$ and $\varphi_{W} \in \widehat{W}$, up to non-zero scalar factors. Fix a face $F \in \Delta^{n-1}$ of dimension $n-r$. As $\widehat{V} \perp_{q_{F}} \widehat{W}$, we in particular have $\varphi_{V} \perp_{q_{F}} \varphi_{W}$. Since $\left(p_{F}\right)_{i}=0$ for all $i \in[n] \backslash F$, this reads

$$
\sum_{i \in F}\left(p_{F}\right)_{i} \varphi_{V}\left(x_{i}\right) \varphi_{W}\left(x_{i}\right)=0 .
$$

Let $P_{1}$ and $P_{2}$ be the two parts of $\mathbb{R} P^{d}$ defined by $H_{V}$ and $H_{W}$.

Consider the two cases:

(1) $H_{V} \neq H_{W}$ : Then the sign of $\varphi_{V}\left(x_{i}\right) \varphi_{W}\left(x_{i}\right)$ determines whether $\left[x_{i}\right]$ lies on $P_{1}$, on $P_{2}$, or both. Thus, from (2.2) and the fact that the $\left(p_{F}\right)_{i}$ are non-negative and not all zero, we deduce that each of the parts contains at least one of the points $\left[x_{i}\right]$ indexed by $F$.

(2) $H_{V}=H_{W}$ : We may and do assume that we chose $\varphi_{V}=\varphi_{W}$. Then the left hand side of (2.2) is a sum of squares. Thus for all (and in particular for at least one) $i \in F$ with $\left(p_{F}\right)_{i}>0$ we get $\varphi_{V}\left(x_{i}\right)=\varphi_{W}\left(x_{i}\right)=0$, which means that $\left[x_{i}\right]$ lies on $H_{V}=H_{W}$.

Thus, $\widehat{W}$ does it. It remains to verify that the general position assumption implies $V \cap W=0$. Assuming the contrary, there is a hyperplane $H$ containing both $V$ and $W$. The assertion for $H_{V}=H_{W}=H$ implies that $H$ contains at least $r$ points from $X$, as well as $V$. This is exactly what we exclude by the general position assumption.

\section{Proof of the Projective Tverberg Theorem}

The proof of Theorem 1.10 is very similar. The main difference is that in place of Theorem 2.1 we invoke the following topological Tverberg theorem, which is due to Bárány et al. [1] in the prime case, and Özaydın [21] and Volovikov [26] in the prime power case.

Theorem 3.1 (Topological Tverberg theorem [1,21,26]) Suppose $r$ is a prime power. If the simplex $\Delta^{n-1}$ of dimension $n-1=(d+1)(r-1)$ is mapped continuously to $\mathbb{R}^{d}$ then the images of some $r$ pairwise disjoint faces $F_{1}, \ldots, F_{r}$ have a point in common. 
Proof of Theorem 1.10 We proceed as in Sect. 2 until we consider the continuous map $f: \Delta^{n-1} \rightarrow \mathbb{R}^{D}(n=(D+1)(r-1)+1)$ that sends a probability distribution $p \in \Delta^{n-1}$ to a subspace $\widehat{W}_{p, \varepsilon} \subset\left(\mathbb{R}^{d+1}\right)^{*}$, considered as an element in $\mathbb{R}^{D}$. Now we apply Theorem 3.1 to $f$ (assuming $r$ is a prime power). This yields $r$ points $p_{1}, \ldots, p_{r} \in \Delta^{n-1}$ with pairwise disjoint supports such that their corresponding subspaces $\widehat{W}_{p_{j}, \varepsilon}$ coincide.

After that we pass to the limit $\varepsilon_{m} \rightarrow 0$, obtaining points $p_{1}, \ldots, p_{r} \in \Delta^{n-1}$ with pairwise disjoint supports (say, $X_{j}:=\operatorname{supp}\left(p_{j}\right)$ ) and a subspace $\widehat{W} \subset\left(\mathbb{R}^{d+1}\right)^{*}$ such that $\widehat{V} \perp_{q_{j}} \widehat{W}$ for all $q_{j}:=q_{p_{j}, 0}, 1 \leq j \leq r$.

The rest of the proof is the same as before, one only has to replace the faces $F$ by the $X_{j}$.

\section{A Transversal Projective Tverberg Theorem}

Tverberg and Vrećica [25] conjectured a common generalization of the ham sandwich theorem, the center transversal theorem, and Tverberg's theorem, which they proved in some special cases. Later Živaljević [30], Vrećica [27], Karasev [15], and Blagojević et al. [4] proved further special cases and colorful extensions.

The following theorem and the subsequent Remark 4.4 generalize all those results (which accumulate to the case $v=d-1$ below) and Theorem 1.10 (which is the special case $m=1$ ).

Theorem 4.1 Let $d>v \geq 0, m \geq 1, d>w:=m(d-v)-1, D:=(d-v)(d-w)$, and let $p$ be a prime. Suppose that $p=2$ or $m=1$ or $d-w$ is even. Let $r_{1}=$ $p^{\alpha_{1}}, \ldots, r_{m}=p^{\alpha_{m}}$ be powers of $p$.

Suppose that $X^{1}, \ldots, X^{m}$ are $m$ point sets in $\mathbb{R} P^{d}$ of size $\left|X^{j}\right|=(D+1)\left(r_{j}-1\right)+1$ and $V \subset \mathbb{R} P^{d}$ is a projective subspace of dimension $v$.

Then there exists a projective subspace $W \subset \mathbb{R} P^{d}$ of dimension $w$ and partitions of each $X^{j}$ into $r_{j}$ pieces respectively,

$$
X^{j}=X_{1}^{j} \dot{\cup} \cdots \dot{\cup} X_{r_{j}}^{j}
$$

such that any pair of hyperplanes $H_{V} \supseteq V$ and $H_{W} \supseteq W$ partitions $\mathbb{R} P^{d}$ into two closed parts so that each part has nonempty intersection with every $X_{i}^{j}$.

Remark 4.2 It is natural to conjecture the same statement also for all positive integers $r_{j}$ and without the technical assumption of $p=2$ or $m=1$ or $d-w$ being even.

Remark 4.3 The assumption that no $r$ points of any $X^{i}$ together with $V$ are contained in a hyperplane will guarantee that $W$ and $V$ intersect transversally.

Remark 4.4 In the case when all $r_{j}=p$, Theorem 4.1 has a colorful extension analogously to the colorful Tverberg-Vrećica theorem of [4]: If for every $j$ we color the point set $X^{i}$ so that every color appears at most $r_{j}-1$ times, then the partitions of the sets $X^{j}$ can be made rainbow colored in the sense that every part $X_{i}^{j}$ uses every color at most once. 
Proof of Theorem 4.1 Let $n_{j}:=\left|X^{j}\right|=(D+1)\left(r_{j}-1\right)+1(1 \leq j \leq m)$. As above we regard $V$ as a linear $(v+1)$-dimensional subspace of $\mathbb{R}^{d+1}$, denoted by $V^{\prime}$. Let $\widehat{V} \subseteq\left(\mathbb{R}^{d+1}\right)^{*}$ be its dual space of dimension $d-v$ (this is the same as going to the projective dual statement of the theorem). We have to find a certain $(w+1)$ dimensional subspace $W^{\prime} \subseteq \mathbb{R}^{d+1}$. We will instead find its dual space $\widehat{W} \subseteq\left(\mathbb{R}^{d+1}\right)^{*}$ of dimension $d-w$.

Note that the dimension of $\widehat{V}$ and $\widehat{W}$ will sum up to $2 d-v-w$. Let $B$ be the set of all $(2 d-v-w)$-dimensional subspaces $U \subseteq\left(\mathbb{R}^{d+1}\right)^{*}$ that contain $\widehat{V}$. Sending $U$ to its quotient $U / \widehat{V}$ gives us a bijection $B \cong G(v+1, d-w)$, which we use to topologize $B$. The Grassmannian here may be considered oriented or not oriented; we defer this choice till the end of the proof.

Let us fix a subspace $U \in B, j \in\{1, \ldots, m\}$ and an $\varepsilon>0$. As in the proof of Theorem 1.10, we want to find $r_{j}$ quadratic forms $q_{p_{i}, \varepsilon}$ on $U$ such that $p_{1}, \ldots, p_{r_{j}} \in$ $\Delta^{n_{j}-1}$ have pairwise disjoint supports and such that the orthogonal complement of $\widehat{V}$ in $U$ with respect to any $q_{p_{i}, \varepsilon}$ is the same $(d-w)$-subspace of $U$. We use the usual configuration space/test map scheme for topological Tverberg-type theorems, see for example Matoušek [19]: First, as in (2.1), we consider the map $\Delta^{n_{j}-1} \rightarrow S_{U}$ that sends $p \in \Delta^{n_{j}-1}$ to the respective orthogonal complement of $\widehat{V}$, where $S_{U}$ is the Schubert cell in $G(2 d-w-v, d-w)$ that consists of all $(d-w)$-subspaces of $U$ that intersect $\widehat{V}$ trivially. Taking the $r_{j}$ 'th power of this map and restricting the domain to $\left(\Delta^{n_{j}-1}\right)_{\Delta(2)}^{r_{j}}$ (the $r_{j}$-fold pairwise deleted product of $\Delta^{n_{j}-1}$, i.e. the union of products $F_{1} \times \ldots \times F_{r}$ of pairwise disjoint faces of $\Delta^{n_{j}-1}$ ), we obtain a so-called test-map

$$
f_{U, j, \varepsilon}:\left(\Delta^{n_{j}-1}\right)_{\Delta(2)}^{r_{j}} \longrightarrow \Sigma_{r_{j}}\left(S_{U}\right)^{r_{j}}
$$

The set of $r_{j}$-tuples of coefficient vectors $\left(p_{i}\right)_{1 \leq i \leq r_{j}}$ with pairwise disjoint support and equal image in $S_{U}$ can be written as the preimage $f_{U, j, \varepsilon}^{-1}\left(\Delta_{\left(S_{U}\right)^{r_{j}}}\right)$ of the thin diagonal $\Delta_{\left(S_{U}\right)^{r_{j}}}:=\left\{\left(x_{1}, \ldots, x_{r_{j}}\right) \in\left(S_{U}\right)^{r_{j}}: x_{1}=\ldots=x_{r_{j}}\right\}$. The symmetric group on $r_{j}$ elements $\Sigma_{r_{j}}$ acts on both sides of (4.1) by permuting the coordinates, which makes the test-map $f_{U, j, \varepsilon}$ in a natural way $\Sigma_{r_{j}}$-equivariant.

Elements $\widehat{W} \in S_{U}$ can be uniquely written as kernels of linear projections $U \rightarrow \widehat{V}$. When choosing an inner product on $U$, those projections are in bijection with maps $U / \widehat{V} \rightarrow \widehat{V}$, which are elements in $(U / \widehat{V})^{*} \otimes \widehat{V}$. Thus,

$$
S_{U} \cong(U / \widehat{V})^{*} \otimes \widehat{V} \cong \mathbb{R}^{D}
$$

where the first isomorphism is natural in $U \in B$ if the inner products on the $U$ 's are consistently the restriction of a fixed inner product on $\mathbb{R}^{d+1}$. The maps $f_{U, j, \varepsilon}$ depend continuously on $U \in B$. Let $\gamma: E(\gamma) \rightarrow B$ denote the tautological vector bundle over $B \cong G(v+1, d-w)$ of rank $d-w$, its fibers being $U / \widehat{V}$. The union of the Schubert cells $S_{U}$ forms in a natural way a vector bundle over $B$ ( $S_{U}$ being the fiber over $U \in B$ ), which by (4.2) is isomorphic to $\left(\gamma^{*}\right)^{\oplus(d-v)} \cong \gamma^{\oplus(d-v)}$.

Hence the collection of maps $f_{U, j, \varepsilon}(U \in B)$ forms a $\Sigma_{r_{j}}$-equivariant bundle map $f_{j, \varepsilon}$ over $B$, 


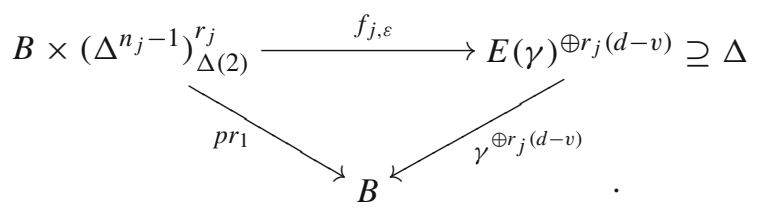

Here, $\Delta \cong E\left(\gamma^{\oplus(d-v)}\right)$ denotes the thin diagonal subbundle, and we are interested in the preimages $f_{j, \varepsilon}^{-1}(\Delta)$. In particular we have to show that

$$
f_{1, \varepsilon}^{-1}(\Delta) \cap \cdots \cap f_{m, \varepsilon}^{-1}(\Delta) \neq \emptyset .
$$

This can be proved exactly as in [15] or [4] using a parametrized Borsuk-Ulam type theorem. The only topological assumption that we need is that the mod- $p$-Euler class of $\Delta, e(\Delta)=e(\gamma)^{d-v} \in H^{D}\left(B ; \mathbb{F}_{p}\right)$, satisfies

$$
e(\Delta)^{m-1} \neq 0
$$

We know that $e(\Delta)^{m-1}=e(\gamma)^{(v+1)-(d-w)} \neq 0$ if $p=2$ and we consider the nonoriented Grassmannian (see Hiller [14]) or if $p>2, \operatorname{dim} \gamma=d-w$ is even, and we consider the oriented Grassmannian (see [15, Lemma 8]). The same is trivially true if $m=1$, since then $B$ is a single point pt and the "Euler class" is a generator of $H^{0}\left(\mathrm{pt} ; \mathbb{F}_{p}\right)$. Finally, (4.4) holds true if $p=2$ or $d-w$ is even or $m=1$.

As in the previous proofs, letting $\varepsilon$ go to zero finishes the proof.

\section{A Projective Center Transversal Theorem}

As an immediate corollary of Theorem 4.1 we obtain a projective generalization of Dol'nikov's [6-8] and Živaljević's [29] center transversal theorem (which is the special case $v=d-1)$ :

Corollary 5.1 (Projective center transversal theorem) Let $d>v \geq 0, m \geq 1, d>$ $w:=m(d-v)-1$, and $D:=(d-v)(d-w)$.

Suppose that $\mu_{1}, \ldots, \mu_{m}$ are $m$ Borel probability measures on $\mathbb{R} P^{d}$ and $V \subset \mathbb{R} P^{d}$ is a projective subspace of dimension $v$.

Then there exists a projective subspace $W \subset \mathbb{R} P^{d}$ of dimension $w$ such that any pair of hyperplanes $H_{V} \supseteq V$ and $H_{W} \supseteq W$ partitions $\mathbb{R} P^{d}$ into two closed parts $P_{1}$ and $P_{2}$ so that for any $j \in\{1, \ldots, m\}$,

$$
\mu_{j}\left(P_{1}\right), \mu_{j}\left(P_{2}\right) \geq \frac{1}{D+1} .
$$

Proof We approximate the measures $\mu_{j}$ by finite point sets $X^{j}$ (as in the proof of Tverberg and Vrećica [25] that their conjecture implies the center transversal theorem). We apply Theorem 4.1, avoiding the parity assumption of $d-w$ being even by simply taking $p=2$ and $r_{j}$ equal to appropriate powers of two. Both parts $P_{1}$ and $P_{2}$ will 
contain at least one point from every $X_{i}^{j}$ and thus at least $r_{j}$ points from every $X^{j}$. Since $r_{j} /\left|X^{j}\right| \geq 1 /(D+1)$ and $G(d+1, w+1)$ is compact, a limit argument finishes the proof.

Remark 5.2 The case $m=1$ could be called a projective center point theorem in analogy with Rado's center point theorem [22], which is the special case $m=1$ and $v=d-1$. Similarly, the case $m=d$ and $v=w=d-1$ is the ham sandwich theorem; compare with Blagojević-K. [2, Theorem 10] for an actual projective mass partition theorem.

\section{Another Projective Tverberg Theorem}

We could also ask what happens if we allow in Theorem 1.10, for example, to choose $V$ arbitrarily. A partial answer is given by the following:

Theorem 6.1 Let $0 \leq v<d, r_{1}$ and $r_{2}$ be the powers of the same prime $p$, and $X^{1}$ and $X^{2}$ be finite point sets with $\left|X^{j}\right|=(D+1)\left(r_{j}-1\right)+1$, where $D:=(d-v)(d-w)$ and $w:=d-v-1$. We further assume that $D$ is even and the binomial coefficient $\left(\left\lfloor\frac{d+1}{2}\right\rfloor\left\lfloor\frac{v+1}{2}\right\rfloor\right)$ is nonzero $\bmod p$.

Then there exist projective subspaces $V, W \subset \mathbb{R} P^{d}$ of dimensions $v$ and $w$ and partitions of every $X^{j}$ into $r_{j}$ pieces

$$
X^{j}=X_{1}^{j} \dot{\cup} \cdots \dot{\cup} X_{r_{j}}^{j}
$$

such that any pair of hyperplanes $H_{V} \supseteq V$ and $H_{W} \supseteq W$ partitions $\mathbb{R} P^{d}$ into two parts so that each part has nonempty intersection with every $X_{i}^{j}$.

Remark 6.2 It was already known to Kummer that $\left(\begin{array}{l}n \\ k\end{array}\right)$ is nonzero $\bmod p$ if and only if for all $i \geq 0$ the $i$ 'th digit of $n$ in the $p$-ary representation is greater or equal to the $i$ 'th digit of $k$.

Remark 6.3 As a corollary we obtain a corresponding center point theorem for partitioning two measures under the assumption that $D$ is even. The proof is analogous to the one of Corollary 5.1 .

Proof of Theorem 6.1 We follow the proof of Theorem 1.10 for $X^{j}$ and describe the set of appropriate $\widehat{W}_{j}$ for every $\widehat{V}$ as a preimage. We have to find some $\widehat{V}$ such that $\widehat{W}_{1}$ and $\widehat{W}_{2}$ are equal. Their difference is an element of $\left(\mathbb{R}^{d+1} / \widehat{V}\right) \otimes \widehat{V}$. So (the formal argument is similar to the proof of Theorem 4.1, using a parameterized testmap) we observe that the spaces $\left(\mathbb{R}^{d+1} / \widehat{V}\right) \otimes \widehat{V}$ form a vector bundle over the Grassmannian $G(d+1, v+1)$ of all possible $V$ and this bundle is isomorphic to $\gamma \otimes \gamma^{\perp}$, where $\gamma$ is the tautological bundle over the Grassmannian and $\gamma^{\perp}$ its orthogonal complement bundle.

It remains to check that the Euler class $e\left(\gamma \otimes \gamma^{\perp}\right) \in H^{D}\left(G(d+1, v+1) ; \mathcal{F}_{p}\right)$ is nonzero (the coefficients are $\mathbb{F}_{p}$ with possible twist corresponding to the orientation 
character of $\left.\gamma \otimes \gamma^{\perp}\right)$. An open neighborhood (and thus the tangent space) of $G(d+$ $1, v+1)$ at a point $\widehat{V}$ can be identified with

$$
\widehat{V}^{*} \otimes \widehat{V}^{\perp}=\operatorname{Hom}\left(\widehat{V}, \widehat{V}^{\perp}\right),
$$

because adding such maps to the inclusion $\widehat{V} \hookrightarrow \mathbb{R}^{d+1}$ gives linear maps $\widehat{V} \rightarrow \mathbb{R}^{d+1}$ with all possible images intersecting $\widehat{V}^{\perp}$ trivially. Thus the images of such maps constitute the Schubert cell neighborhood of $\widehat{V}$. Since the identifications are natural and $\gamma^{*} \cong \gamma$, we have

$$
\gamma \otimes \gamma^{\perp} \cong T G(d+1, v+1)
$$

Thus the Euler class $e\left(\gamma \otimes \gamma^{\perp}\right)$ is Poincaré dual to the element of $H_{0}(G(d+1, v+$ $\left.1) ; \mathbb{F}_{p}\right)$ equal to the Euler characteristic of $G(d+1, v+1) \bmod p$; the cohomology twist is eliminated because it has to be tensor multiplied by the orientation sheaf of $T G(d+1, v+1)$. The Euler characteristic of the Grassmannian is equal to the value of the $q$-binomial coefficient $\left(\begin{array}{l}d+1 \\ v+1\end{array}\right)_{q}$ at $q=-1$. This value can be calculated by the inductive formula:

$$
\left(\begin{array}{l}
n \\
k
\end{array}\right)_{-1}=\left(\begin{array}{l}
n-1 \\
k-1
\end{array}\right)_{-1}+(-1)^{k}\left(\begin{array}{l}
n-1 \\
k
\end{array}\right)_{-1}, \quad\left(\begin{array}{l}
n \\
0
\end{array}\right)_{-1}=\left(\begin{array}{l}
n \\
n
\end{array}\right)_{-1}=1,
$$

and it finally turns out to be zero if $D$ is odd and equal to

$$
\left(\begin{array}{l}
\left\lfloor\frac{d+1}{2}\right\rfloor \\
\left\lfloor\frac{v+1}{2}\right\rfloor
\end{array}\right)
$$

if $D$ is even.

\section{A Corresponding “Transversal” Generalization}

In this section we extend Theorem 6.1 under some algebraic assumptions to the case of more than two masses in the same way as Theorem 4.1 extends Theorem 1.10. The pay-off for establishing this is that the dimension of $V$ and $W$ must increase.

Let $0 \leq v, w<d, m \geq 1, r_{j}=2^{\alpha_{j}}(1 \leq j \leq m), \widehat{v}:=d-v, \widehat{w}:=d-w, D:=\widehat{v} \widehat{w}$. Suppose that $X^{1}, \ldots, X^{m}$ are $m$ point sets in $\mathbb{R} P^{d}$ of size $\left|X^{j}\right|=(D+1)\left(r_{j}-1\right)+1$. We will find a condition under which there exist projective subspaces $V, W \subset \mathbb{R} P^{d}$ of dimensions $v$ and $w$ and partitions of every $X^{j}$ into $r_{j}$ pieces

$$
X^{j}=X_{1}^{j} \dot{\cup} \cdots \dot{\cup} X_{r_{j}}^{j},
$$

such that any pair of hyperplanes $H_{1} \supseteq V$ and $H_{2} \supseteq W$ partitions $\mathbb{R} P^{d}$ into two parts so that each part has nonempty intersection with every $X_{i}^{j}$.

Similarly to the above sections, the topological setup for this is the following. Let $B:=F(\widehat{v}, \widehat{v}+\widehat{w}, d+1)$ be the partial flag manifold of all pairs $(\widehat{V}, U)$ of linear 
subspaces of $\mathbb{R}^{d+1}$ such that $\operatorname{dim}(\widehat{V})=\widehat{v}, \operatorname{dim} U=\widehat{v}+\widehat{w}$, and $\widehat{V} \subset U$. Let $\eta$ be the rank $D$ vector bundle over $B$ whose fiber over $(\widehat{V}, U)$ is $\widehat{V} \otimes(U / \widehat{V})$. Let $w_{i}(\eta) \in H^{i}\left(B ; \mathbb{F}_{2}\right)$ denote its Stiefel-Whitney classes. The condition we look for is

$$
w_{D}(\eta)^{m-1} \neq 0
$$

We can put this condition into purely algebraic terms as follows using a particular instance of the splitting principle. Let $F:=\operatorname{Flag}\left(\mathbb{R}^{d+1}\right)=F(1,2, \ldots, d+1)$ be the complete real flag manifold in $\mathbb{R}^{d+1}$. There is a canonical projection $p: F \rightarrow B$. Let $\ell_{1}, \ldots, \ell_{d+1}$ be the tautological line bundles over $F$. Their first Stiefel-Whitney classes $e_{i}:=w_{1}\left(\ell_{i}\right)$ generate the ring $H^{*}\left(F ; \mathbb{F}_{2}\right)$. More exactly,

$$
H^{*}\left(F ; \mathbb{F}_{2}\right) \cong \mathbb{F}_{2}\left[e_{1}, \ldots, e_{d+1}\right] /\left(\prod\left(1+e_{i}\right)=1\right),
$$

where $\left(\prod\left(1+e_{i}\right)=1\right)$ denotes the homogeneous ideal generated by the elementary symmetric polynomials in $e_{1}, \ldots, e_{d+1}$. The relation means geometrically that the sum $\ell_{1} \oplus \ldots \oplus \ell_{d+1}$ is trivial. The reader may find (7.2) and other standard facts about vector bundles and their characteristic classes in [18].

Now,

$$
\begin{aligned}
w\left(p^{*} \eta\right) & =w\left(\left(\ell_{1} \oplus \ldots \oplus \ell_{\widehat{v}}\right) \otimes\left(\ell_{\widehat{v}+1} \oplus \ldots \oplus \ell_{\widehat{v}+\widehat{w}}\right)\right) \\
& =\prod_{i, j}^{*} w\left(\ell_{i} \otimes \ell_{j}\right)=\prod_{i, j}^{*}\left(1+w_{1}\left(\ell_{i}\right)+w_{1}\left(\ell_{j}\right)\right),
\end{aligned}
$$

where $\prod_{i, j}^{*}$ denotes a product that runs over all pairs $(i, j)$ with $1 \leq i \leq \widehat{v}$ and $\widehat{v}+1 \leq j \leq \widehat{v}+\widehat{w}$. In particular,

$$
w_{D}\left(p^{*} \eta\right)=\prod_{i, j}^{*}\left(e_{i}+e_{j}\right)
$$

Thus (7.1) is equivalent to $\prod_{i, j}^{*}\left(e_{i}+e_{j}\right)^{m-1} \neq 0$ in $H^{*}\left(F ; \mathbb{F}_{2}\right)$. This proves:

Theorem 7.1 Let $0 \leq v, w<d, m \geq 1, r_{j}=2^{\alpha_{j}}(1 \leq j \leq m), D:=(d-v)(d-w)$, such that the product (7.3) is non-zero in (7.2).

Suppose that $X^{1}, \ldots, X^{m}$ are $m$ point sets in $\mathbb{R} P^{d}$ of size $\left|X^{j}\right|=(D+1)\left(r_{j}-1\right)+1$.

Then there exist projective subspaces $V, W \subset \mathbb{R} P^{d}$ of dimensions $v$ and $w$ and partitions of every $X^{j}$ into $r_{j}$ pieces

$$
X^{j}=X_{1}^{j} \dot{\cup} \cdots \dot{\cup} X_{r_{j}}^{j}
$$

such that any pair of hyperplanes $H_{1} \supseteq V$ and $H_{2} \supseteq W$ partitions $\mathbb{R} P^{d}$ into two parts so that each part has nonempty intersection with every $X_{i}^{j}$.

Remark 7.2 A standard argument for proving that a polynomial $p$ does not lie in an ideal $I$ is to present a geometric point in the associated variety $V(I)$ on which the 
regular function $p$ does not vanish. However in our situation this trick does not work: The projective variety defined by $I:=\left(\prod\left(1+e_{i}\right)=1\right)$ (we equal every homogeneous component separately) has no geometric point at all, which can be easily checked. That is, the radical of the homogeneous ideal generated by the homogeneous components of $\prod\left(1+e_{i}\right)$ of positive degree in $\overline{\mathbb{F}}_{2}\left[e_{1}, \ldots e_{d+1}\right]$ contains already all homogeneous polynomials of positive degree. Besides, it is already clear that $w_{D}(\eta)^{m-1}$ must vanish in $H^{*}\left(F ; \mathbb{F}_{2}\right)$ for large $m$, since Theorem 7.1 cannot hold for arbitrary large $m$.

Acknowledgments The research of Karasev was supported by the Dynasty foundation. The research of Matschke was supported by NSF Grant DMS-0635607, by an EPDI fellowship, and by MPIM Bonn. We thank Karim Adiprasito, Pavle Blagojević, Moritz Firsching, Peter Landweber, Louis Theran, and Günter Ziegler for useful discussions.

\section{References}

1. Bárány, I., Shlosman, S.B., Szúcs, A.: On a topological generalization of a theorem of Tverberg. J. London Math. Soc. 23, 158-164 (1981)

2. Blagojević, P.V.M., Karasev, R.N.: Extensions of theorems of Rattray and Makeev. (2010). http://arxiv. org/abs/1011.0869

3. Blagojević, P.V.M., Matschke, B., Ziegler, G.M.: Optimal bounds for the colored Tverberg problem. (2009). http://arxiv.org/abs/0910.4987

4. Blagojević, P.V.M., Matschke, B., Ziegler, G.M.: Optimal bounds for a colorful Tverberg-Vrećica type problem. Adv. Math. 226, 5198-5215 (2011)

5. Deo, S.: Index of a finitistic space and a generalization of the topological central point theorem. J. Ramanujan Math. Soc. 28(2), 223-232 (2013)

6. Dol'nikov, V.L.: Common transversals for families of sets in $\mathbb{R}^{n}$ and connections between theorems of Helly and Borsuk. Dokl. Akad. Nauk USSR 297(4), 777-780 (1987). In Russian

7. Dol'nikov, V.L.: A generalization of the ham sandwich theorem (In Russian). Mat. Zametki 52(1993), 27-37 (1992). English translation in Math. Notes 52(1993), 771-779

8. Dol'nikov, V.L.: Transversal of families of sets in $\mathbb{R}^{n}$ and a connection between the Helly and Borsuk theorems. Russ. Acad. Sci. Sbornik Math. 79(1), 93-107 (1994)

9. Engström, A.: A local criterion for Tverberg graphs. Combinatorica 31(3), 321-332 (2011)

10. Engström, A., Norén, P.: Tverberg's theorem and graph coloring. (2011). http://arxiv.org/abs/1105. 1455

11. Grünbaum, B.: Partitions of mass-distributions and of convex bodies by hyperplanes. Pac. J. Math. 10, $1257-1261(1960)$

12. Hell, S.: On the number of Tverberg partitions in the prime power case. Eur. J. Comb. 28(1), 347-355 (2007)

13. Hell, S.: Tverberg's theorem with constraints. J. Comb. Theory A 115(8), 1402-1416 (2008)

14. Hiller, H.L.: On the cohomology of real Grassmannians. Trans. Amer. Math. Soc. 257, 521-533 (1980)

15. Karasev, R.N.: Tverberg's transversal conjecture and analogues of nonembeddability theorems for transversals. Discrete Comput. Geom. 38, 513-525 (2007)

16. Karasev, R.N.: Dual theorems on central points and their generalizations. Sbornik Math. 199(10), 1459-1479 (2009). Corrected English version available at http://arxiv.org/abs/0909.4915

17. Karasev, R.N.: A topological central point theorem. Topol. Appl. 159(3), 2012, 864-868. http://arxiv. org/abs/1011.1802

18. Luke, G., Mishchenko, A.S.: Vector Bundles and Their Applications. Springer, New York (1998)

19. Matoušek, J.: Using the Borsuk-Ulam Theorem, 2nd edn. Springer, Berlin (2007)

20. Neumann, B.H.: On an invariant of plane regions and mass distributions. J. Lond. Math. Soc. 20, 226-237 (1945)

21. Özaydın, M.: Equivariant maps for the symmetric group. Preprint 17, 155-165 (1987)

22. Rado, R.: A theorem on general measure. J. Lond. Math. Soc. 21, 291-300 (1947)

23. Stong, R.E.: Cup products in Grassmannians. Topol. Appl. 13, 103-113 (1982)

24. Tverberg, H.: A generalization of Radon's theorem. J. Lond. Math. Soc. 41, 123-128 (1966) 
25. Tverberg, H., Vrećica, S.: On generalizations of Radon's theorem and the ham sandwich theorem. Eur. J. Comb. 14, 259-264 (1993)

26. Volovikov, AYu.: On a topological generalization of the Tverberg theorem. Math. Notes 59(3), 324-326 (1996)

27. Vrećica, S.: Tverberg's conjecture. Discrete Comput. Geom. 29, 505-510 (2003)

28. Vućić, A., Živaljević, R.T.: Notes on a conjecture of Sierksma. Discrete Comput. Geom. 9, 339-349 (1993)

29. Živaljević, R.T., Vrećica, S.T.: An extension of the ham sandwich theorem. Bull. Lond. Math. Soc. 22, 183-186 (1990)

30. Živaljević, R.T.: The Tverberg-Vrećica problem and the combinatorial geometry on vector bundles. Isr. J. Math. 111, 53-76 (1999) 